had to drive seven Kenyans back to their hotel. Having just won some gold medals they were in good spirits and speaking loudly in Swahili which developed into singing. I had no idea what they were singing but it was so lovely and melodic. They insisted that I must visit Kenya and told me there are plenty of teeth to see in their country!

The first of August came round quickly, so I hung up my volunteer uniform and reached for the scrub top. The new job at Glasgow Dental Hospital meant new faces, new roles, lots of paperwork, new computer systems, and the thought of anxious children, de-bonded brackets and modules. What had I done? General practice hadn't been that bad!

My timetable revealed I would have a split week, spending time between the Dental Hospital and the Yorkhill Children's Hospital in Glasgow. Dental hospital days would include ortho emergencies, paediatric trauma clinics and A\&E sessions, inhalation sedation and consultant clinics. My Yorkhill days would include GA sessions, treatment sessions and caring for those children on chemotherapy with mucositis. Gosh, the learning curve appeared steep, but I do like a challenge.

In between these sessions, colleagues had learnt about my love of the bicycle and had informed me they wanted to purchase one; could I offer advice? Fleur the dentist moonlighted as Fleur the bicycle advisor. I could get used to it.

My first session on clinic with Professor Welbury was inspirational. A 9-year-old had been fighting with her scooter and the upper centrals lost. Professor Welbury supervised me repositioning the upper centrals and splinting them: all in a day's work in paediatric dentistry.

I am learning fast. There is talk of audits, case reports, poster presentations and the forthcoming International Association of Paediatric Dentistry (IAPD) meeting in Glasgow in 2015. I get the feeling I'm going to be busy, but as I said I not only like a challenge, but believe I will thrive on it.

\title{
DENTAL PROFESSIONALS CAN MAKE HUGE DIFFERENCE TO HOMELESS
}

The Queen's Nursing Institute (QNI) is encouraging dental professionals to join their Homeless Health Network and help to improve oral healthcare for people experiencing homelessness.

Project Manager David ParkerRadford told the BDJ: 'People experiencing homelessness are an extra high needs group for dental care. One Community Dental Service in London opened up access to dental care for homeless people and reported that $99 \%$ of those seen needed treatment. Poor oral health can stem from lack of a toothbrush, substance use, poor diet, mental health conditions, and lack of good dental hygiene awareness. The QNI believes the dental profession can play a hugely significant role in supporting health improvement for homeless people, through offering tailored accessible services, respectful staff, supportive self-care advice and access to a simple and cheap health improvement tool - a toothbrush. In fact it is one of the areas where the greatest strides in overall population oral health improvement can be made'

The Homeless Health Network is free to join and network members also receive six issues of Homeless Health News each year. By joining the network you can participate in consultations, access e-learning and help be part of a movement of health professionals calling for better healthcare for the homeless.

To join email david.parker-radford@qni.org.uk or visit http://www.qni.org.uk/for_nurses/homeless_health.

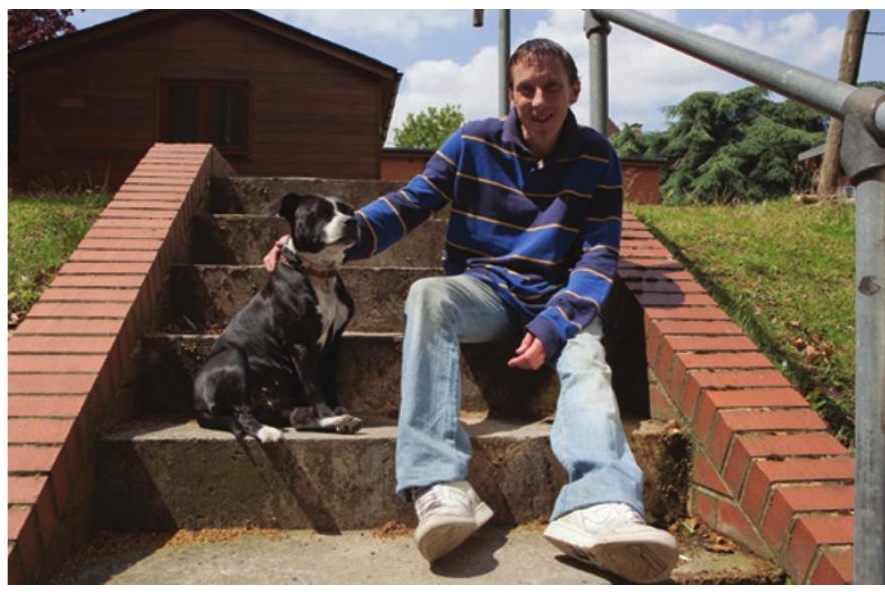

\section{BOOK REVIEW}

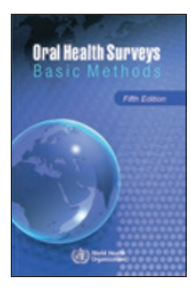

ORAL HEALTH SURVEYS: BASIC METHODS, 5TH EDITION

WHO

World Health Organization

price $€ 25.00 ;$ pp 125

ISBN 9789241548649

This manual aims to encourage national oral health survey planners to standardise measurements of oral diseases and conditions that are important for planning and evaluation of oral health programmes, as well as to ensure the comparability of data collected in a wide range of environments. It does this by applying the WHO global approach to chronic disease surveillance to an operational model for integration of oral health into chronic disease surveillance systems.

This manual is now in its fifth edition. Since the fourth edition, the WHO has developed new tools for implementation of oral health surveys. In addition to guidelines for clinical examination of oral health status, instruments have been developed for surveillance of self-reported oral health and related risk factors. Due to the shared major risk factors of non-communicable chronic and oral diseases, the WHO STEPwise approach to surveillance (STEPS) facilitates the inclusion of oral health in national surveillance systems for non-communicable chronic diseases.

Section one of the manual provides background information for survey planners on the methods and approaches relevant to collection of clinical data on oral health status. Section two covers self-reporting of oral health and the risk factors assessed by means of questionnaires. The final section describes survey activity, such as data analysis and survey reporting.

In the acknowledgements section, it says the web-based version of this manual is available from the oral health page of the WHO website. The online version can also be accessed from www.icd.org/content/publications/ WHO-Oral-Health-Surveys-BasicMethods-5th-Edition-2013.pdf. This is due to the financial support from the Philip Dear Foundation, the European section of the International College of Dentists.

This manual is essential reading for all dentists wishing to undertake oral health surveys. It is a practical guide and very useful. It is clearly and concisely written. Fifteen pages of 71 colour plates are included to enhance the text.

This new publication will facilitate education within dental public health and easy access to the online version of the manual will be of great help for survey implementation in developing countries.

C. A. YEUNG 